\title{
Thermographic analysis of building structures by passive transient measurements
}

\author{
by R. Olbrycht
}

* Lodz Univ. of Technology, Inst. of Electronics, 93-005, 211/215 Wolczanska Str., Lodz, Poland, robert.olbrycht@p.lodz.pl

\begin{abstract}
The investigation of buildings with thermographic cameras often requires certain external conditions, depending on the required effect. When the desired effect is the localisation of thermal bridges, it is advised to carry on the measurements during the night. This paper demonstrates how the results may change depending on the time of day.

\section{Introduction}

Thermal imaging cameras are often used as a tool to investigate the quality of buildings' thermal isolation. If there are lacking parts of this insulation within the wall structure, it causes heat losses. This problem may be mitigated during summer, but in winter there is often significant difference of indoors and outdoors temperature. It causes heat flow from inside to outside, resulting in warmer areas seen in thermal images. These areas, called thermal bridges [1], are the cause of increased heating costs [2]. This is, however, not the only problem, because locally increased temperature outside of the wall often causes local decrease inside, leading to the presence of dampness and fungi. Due to the above mentioned reasons, the best conditions for thermal bridges localisation occurs during winter and in the night, before sunrise. Sun needs to be avoided, as it can locally heat up the surface making the investigation impossible. This paper investigates the influence of the time of the day on the performance of thermographic building investigation, including also detection of mured areas (e.g. where windows were removed and gaps filled with bricks).
\end{abstract}

\section{Measurement configuration}

The author performed an exemplary thermographic investigation lasting 24 hours. The configuration used during the experiment is shown in Fig. 1 , where the examined building is seen by the Jenoptik VarioCam ${ }^{\circledR}$ HiRes thermal imaging camera from the distance of 90 meters. The camera is located 18 meters above the ground level while the building's height is 6 meters. In this configuration, during the $24 \mathrm{~h}$ period, the front wall of the investigated building is never directly illuminated by the sun, according to figure 1 . The experiment took place in March, in Lodz (Poland) with the sunrise on 05:49 and sunset on 17:53 and the camera automatically took pictures every 30 minutes in parallel with the visual camera (Nikon D5100). Ambient temperature and relative humidity varied from $-3{ }^{\circ} \mathrm{C} / 74 \%$ in the night to $10^{\circ} \mathrm{C} / 27 \%$ in the afternoon, with pressure about $1035 \mathrm{hPa}$. Prevailing ESE wind speed not exceeded $13 \mathrm{kts}$ during the day and diminished to near zero in the night. Sky was clear, without clouds or fog. Estimated visibility was about $10 \mathrm{~km}$.

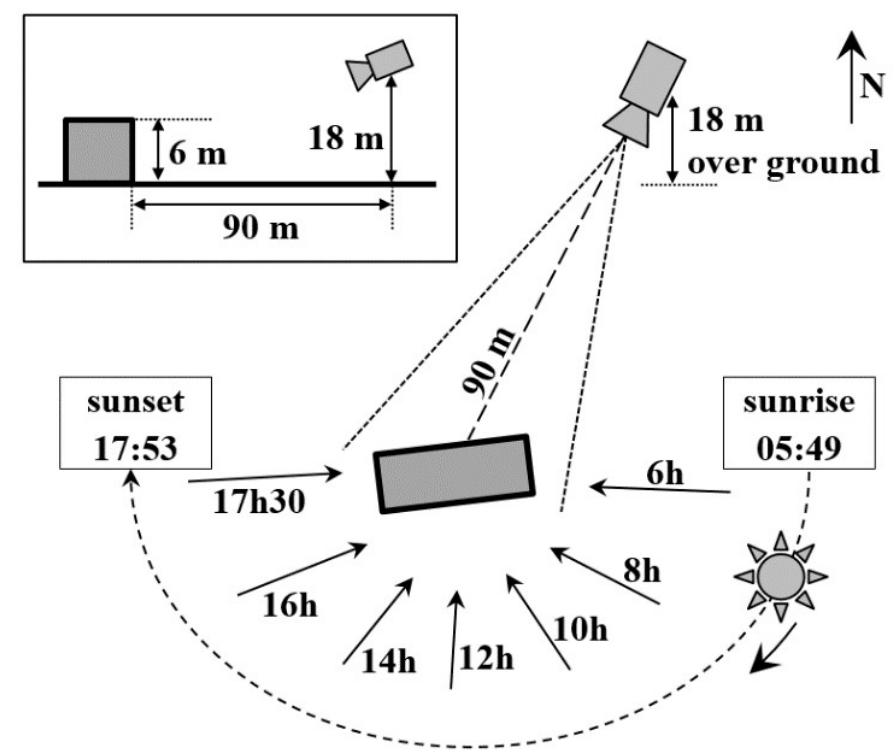

Fig. 1. The configuration used during the experiment on March 


\section{Measurement results}

The exemplary pictures of the investigated building taken at $5 \mathrm{AM}, 7: 30 \mathrm{AM}$ and 11:30 AM are shown in Fig. 2a, $3 a$ and $4 a$, respectively. Corresponding thermal images are shown in Fig. $2 b, 3 b$ and $4 b$, respectively. During the experiment, such image pairs were acquired every 30 minutes, giving 48 visual and 48 thermal images for further investigation. Let us note, that during this 24 hour acquisition period, the imaged wall of the building was never directly heated by sun rays, as shown in Fig. 1. Hence a question arises, whether it is required to investigate the building at night or it is possible to obtain satisfactory results during the daytime. This analysis is performed in this paper, taking into consideration both detection of thermal bridges (visible in the right part of the building) and mured areas (visible in the upper, central part of the wall). Interestingly, these thermal bridges can be observed both in Fig. $2 \mathrm{~b}$ and $3 \mathrm{~b}$ but not in Fig. $4 b$, while mured areas are visible in both Fig. $2 b$ and $4 b$, but tend to disappear in Fig. $3 b$.

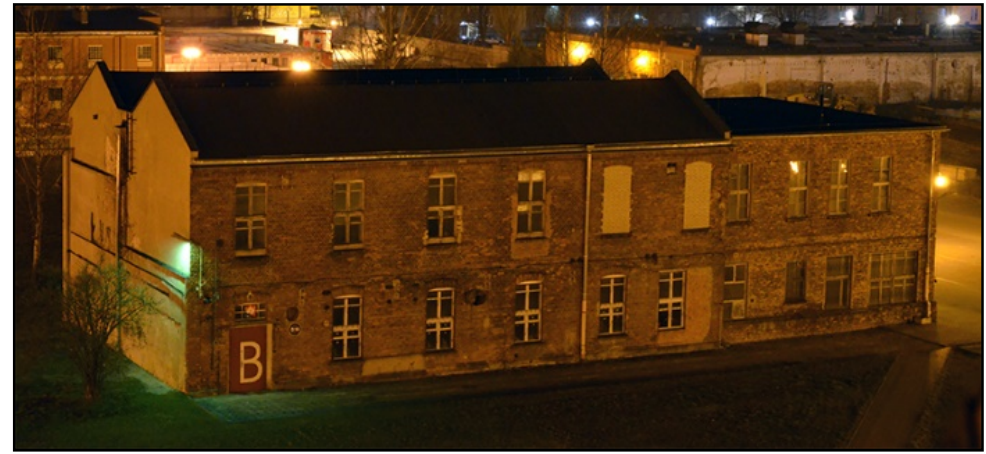

a)

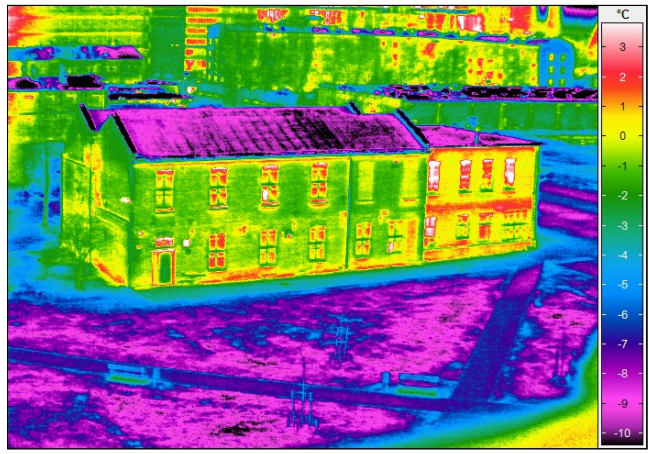

b)

Fig. 2. a) Visual image and b) thermal image of the investigated building, acquired at 5:00

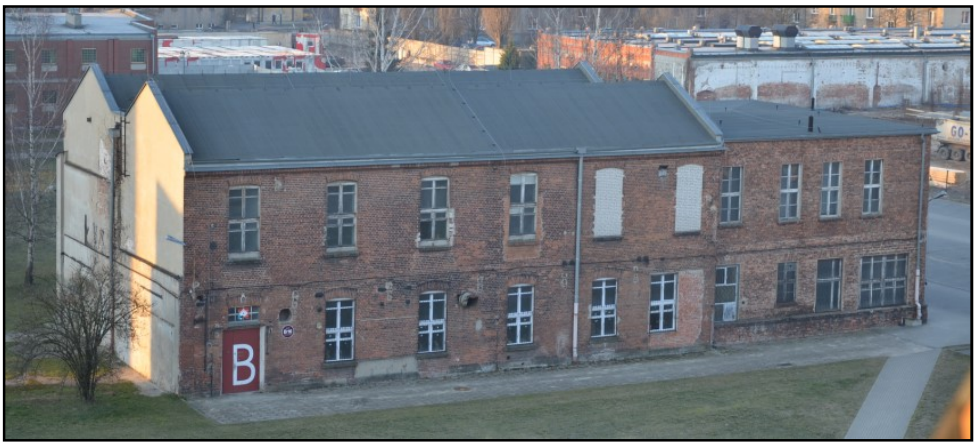

a)

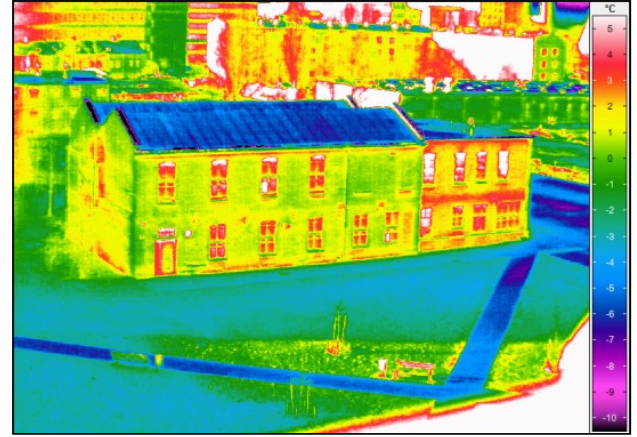

b)

Fig. 3. a) Visual image and b) thermal image of the investigated building, acquired at 7:30

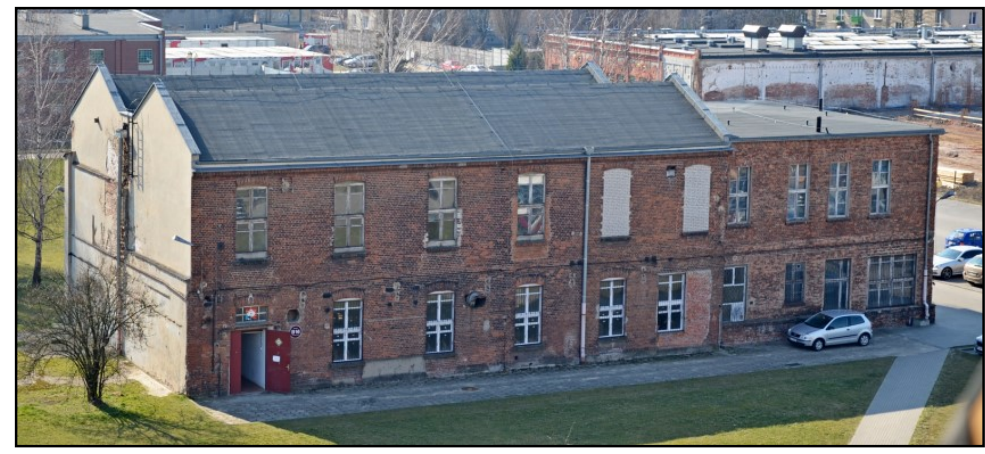

a)

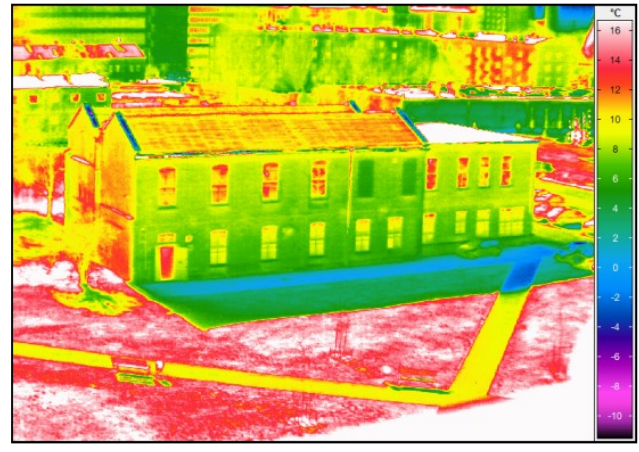

b)

Fig. 4. a) Visual image and b) thermal image of the investigated building, acquired at 11:30 


\section{Analysis of the acquired data}

The same sequence of 48 acquired thermal images was used both for thermal bridges and mured areas detection analysis. It was done by selecting regions of interest (ROI) and taking average temperature value for each ROI, separately for every frame, creating plots of temperature value within the 24 hour time period. This averaging is a standard approach enabling noise suppression, leading to smoother temperature plots.

\subsection{Thermal bridges}

In the acquired thermal images, two small ROI were marked (Fig. 5a), corresponding to wall surface and thermal bridge. Next, temperature plots from these ROI were created (Fig. $5 \mathrm{~b}$ ). It is clearly seen that the highest difference between these plots occurs between $5 \mathrm{AM}$ and $6 \mathrm{AM}$. It corresponds to the highest thermal contrast and highest probability of thermal bridge notification. In the noon, the thermal contrast is 4 times worse, reaching $0.5^{\circ} \mathrm{C}$. One needs to remember that during this experiment the sun was not illuminating directly the investigated wall. With direct sun illumination, the wall temperature would increase significantly, with local temperature differences caused by changes of surface and sub-surface thermal properties of materials, in the same time diminishing the chances of correct interpretation of the acquired thermal data. Based on the research results it is advised to carry on the measurements in the hours preceding the sunrise. More information about building inspection regarding the thermal insulation can be found in e.g. [3-4]. To avoid false interpretation of measurement results, wall corners should be considered as described in [5]. Nonetheless, the most essential factor for thermal bridges localisation is the thermal contrast, as discussed above.

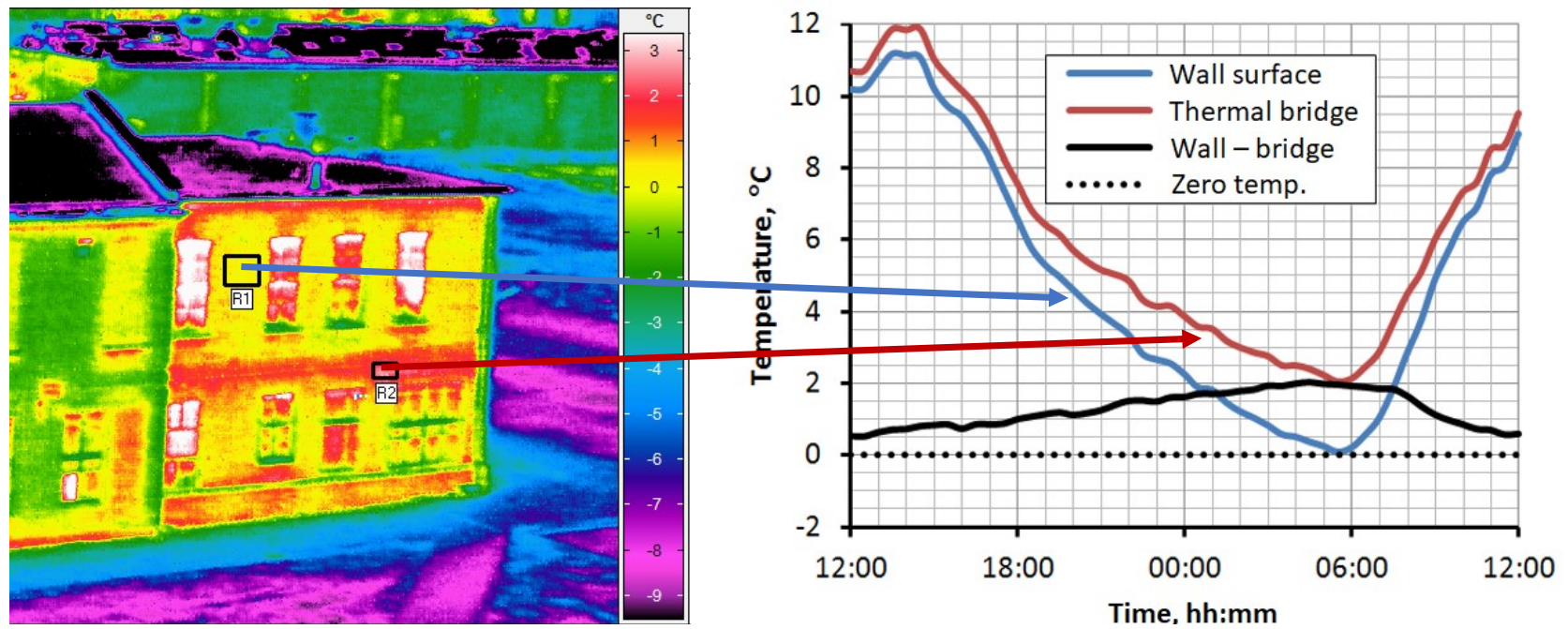

Fig. 5. a) Regions of interest marked in the thermal image,

b) corresponding time plots of mean temperature values from ROIs.

\subsection{Mured areas}

In case of mured areas, they are clearly visible for naked eye in the inspected building, but in practice it is often not the case. Detection of such areas may be desired especially in old buildings belonging to the cultural heritage, subjected to renovations in the past. Such detection is possible thanks to transient measurements, but a question arises, what is the best period of time to perform it. To answer this question, similarly as in previous case, two small ROI were marked in Fig. 6a. Next, temperature plots from these ROI were created (Fig. 6b). In this case, the calculated difference between them reaches its maximum (about $1.5^{\circ} \mathrm{C}$ ) in the night-time, starting after the sunset and lasting to the sunrise. This contrast diminishes substantially in the morning and evening, reaching zero about 8 AM and 4 PM. Interestingly, about the noon, this contrast reaches about $1{ }^{\circ} \mathrm{C}$, however the difference is opposite than in the night-time. As thermal contrast rarely takes high values, it is advised to use camera with low value of NETD (Noise Equivalent Temperature Difference), to maintain low level of noise with such narrow spans.

The technique using the Sun as the natural source of thermal excitation is referred to as solar loading. The Sun causes temperature increase at the surface of inspected walls, which is dependent on the wall sub-surface structure [6]. Due to this structure, there can appear thermal patterns at the surface [7], that reveal this structure. In case of examination indoors, one can use artificial sources of heat, e.g. infrared lamps or flashlights [8]. For even better quality of results, one can use pulse-phase or lock-in technology, to convert acquired sequence of thermal images from time to frequency domain [9]. Nevertheless, for outdoor inspection of walls not directly illuminated with the Sun, night is the optimal time to look for mures. 

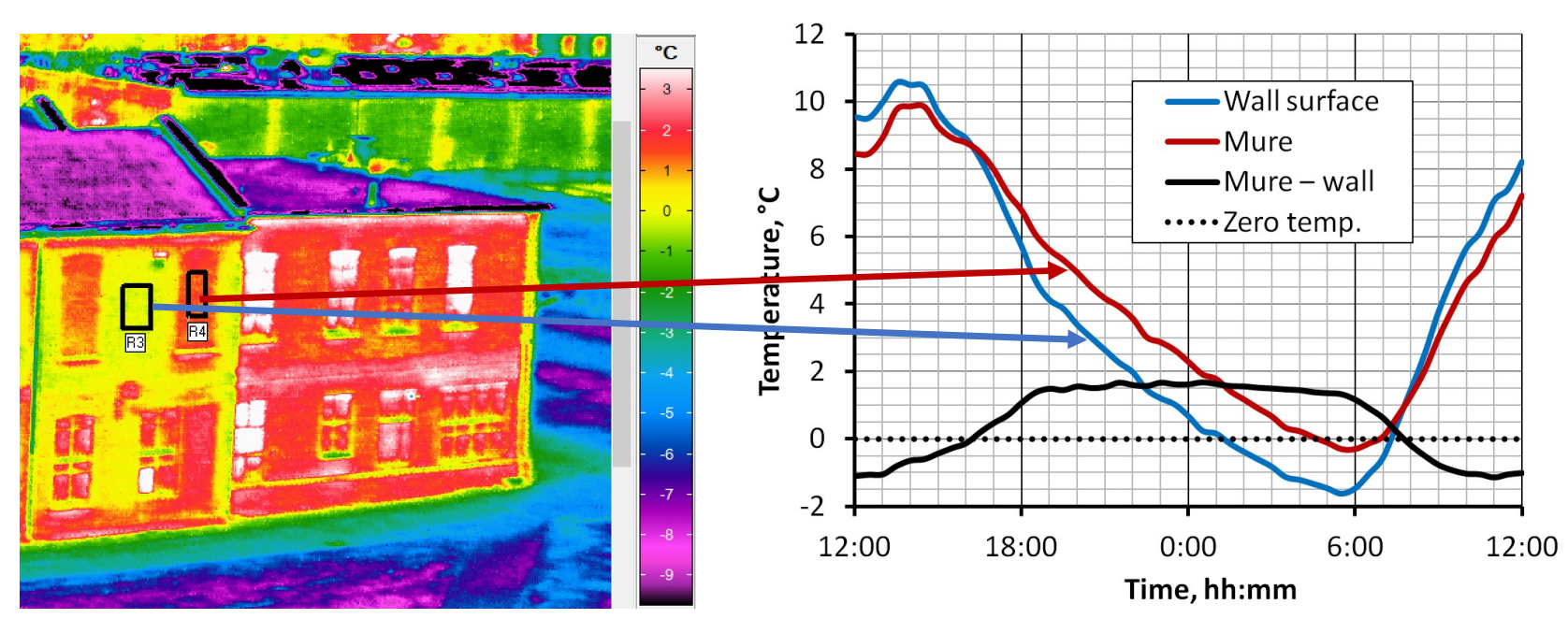

Fig. 6. a) Regions of interest marked in the thermal image,

b) corresponding time plots of mean temperature values from ROIs.

\section{Conclusions}

Infrared thermal imaging is often applied for thermal bridges detection. To maintain the thermal contrast at possibly high level during measurements, it is advised to perform it during the night, before the sunrise. The conducted research revealed that after the sunset this contrast increase is nearly linear with time. The worst time for thermal bridges detection is about noon. When mures are to be localized instead of thermal bridges, but the wall is not directly illuminated with sun, the best results are also possible during the night. In this case, however, according to performed measurements, the contrast reaches plateau between the sunset and sunrise. Noon is also acceptable, but with lower contrast. Mornings as well as evenings should be avoided, as the contrast is near zero in these periods. One should take into consideration, however, that different measurement conditions can influence the optimal time for performing these measurements.

\section{REFERENCES}

[1] Dumitrescu L., Baran I., Aurel Pescaru R., "The Influence of Thermal Bridges in the Process of Buildings Thermal Rehabilitation", Procedia Engineering, Vol. 181, pp. 682-689, 2017, ISSN 1877-7058.

[2] Kuusk K., Kurnitski J., Kalamees T., "Calculation and compliance procedures of thermal bridges in energy calculations in various European countries", Energy Procedia, Vol. 132, pp. 27-32, 2017, ISSN 1876-6102.

[3] Wróbel A., Thermography in Documentation and Measurements of Buildings, Dissertations, Wydawnictwa AGH, 2010, ISBN 978-83-7464-309-2

[4] Alhawari A., Mukhopadhyaya P., Thermal bridges in building envelopes - An overview of impacts and solutions (2018), International Review of Applied Sciences and Engineering IRASE, 9(1), 31-40.

[5] Smusz R., Korzeniowski M., Experimental investigation of thermal bridges in building at real conditions, E3S Web Conf. 70 03013, 2018, DOI: 10.1051/e3sconf/20187003013

[6] Washer G., Fenwick R., Bolleni N., Effects of Solar Loading on Infrared Imaging of Subsurface Features in Concrete (2010), Journal of Bridge Engineering - J BRIDGE ENG. 15. 10.1061/(ASCE)BE.1943-5592.0000117.

[7] Ibarra-Castanedo C., Sfarra S., Klein M., Maldague X., Solar loading thermography: Time-lapsed thermographic survey and advanced thermographic signal processing for the inspection of civil engineering and cultural heritage structures (2017), Infrared Physics \& Technology, Volume 82, Pages 56-74, ISSN 1350-4495, https://doi.org/10.1016/j.infrared.2017.02.014.

[8] Kylili A., Fokaides P. A., Christou P., Kalogirou S. A., Infrared thermography (IRT) applications for building diagnostics: A review (2014), Applied Energy, Volume 134, Pages 531-549, ISSN 0306-2619, https://doi.org/10.1016/j.apenergy.2014.08.005.

[9] Moropoulou A., Avdelidis N., Karoglou M., Delegou E., Alexakis E., Keramidas V., Multispectral Applications of Infrared Thermography in the Diagnosis and Protection of Built Cultural Heritage (2018). Applied Sciences. 8. 284. 10.3390/app8020284. 